\title{
Currículos de Computação: porque permanecem assim?
}

\author{
Miguel Jonathan \\ Departamento de Ciência da Computação /IMAT e Programa de História das Ciências e \\ das Técnicas e Epistemologia (HCTE) - Universidade Federal do Rio de Janeiro (UFRJ) \\ Rua Sambaíba, 478 ap.401 22450-140 - Rio de Janeiro - RJ - Brasil \\ migjonathanegmail. com
}

\begin{abstract}
Undergraduate Computer Science programs in Brazilian universities have been historically focused in mathematics, physics and computing science and technology subjects. Mandatory content of inter or multidisciplinary nature designed to give the graduates an awareness on the interactions of these technologies and systems with society represent less than 5\% of the total. In contrast, computing job opportunities in Brazil are turning increasingly to build applications that directly or indirectly affect the lives of people, individually and in society. This article analyzes the reasons for the persistence of this training model 40 years after its introduction.
\end{abstract}

Resumo. A formação universitária em Ciência da Computação no Brasil tem sido focada historicamente em matemática, física e ciência e tecnologia da computação. Conteúdos obrigatórios de natureza inter ou multidisciplinar destinados a preparar o egresso para refletir sobre a interação dessas tecnologias e sistemas com a sociedade representam menos de 5\% do total. Em contraste, as oportunidades de trabalho em computação no Brasil se voltam cada vez mais para a construção de aplicações que afetam direta ou indiretamente a vida das pessoas, individualmente e em sociedade. $O$ artigo analisa os motivos da persistência desse modelo de formação 40 anos após a sua introdução.

\section{Introdução}

Neste ensaio, abordamos os currículos dos cursos de graduação em ciência da computação, como surgiram e evoluíram, com atenção especial para as redes de atores que contribuíram para a conservação, ao longo dos últimos 40 anos, de um formato e conteúdo aproximadamente semelhantes nas diversas universidades brasileiras, apesar das profundas transformações que ocorreram nas relações da computação com quase todos os ramos da atividade humana nesse período.

O texto é uma reflexão crítica sobre a continuidade da absoluta predominância de conteúdos técnicos e científicos nos currículos em face das crescentes responsabilidades dos egressos com as transformações organizacionais, culturais e econômicas que a sua atividade vem promovendo ao longo das últimas décadas.

Seguindo uma metodologia inspirada nos estudos de ciência, tecnologia e sociedade (CTS), em particular a teoria ator-rede [Latour 2007], procuramos seguir os passos dos atores que produziram os currículos, as circunstâncias que contribuíram para as decisões, 
as alianças que se formaram para produzir consenso em torno de uma forma final, e as condições que permitem uma relativa estabilização dos conteúdos.

Nas seções a seguir apresentamos um histórico resumido da formação dos profissionais de computação no Brasil, a partir da introdução dos primeiros computadores, a criação de cursos de tecnologia pela iniciativa da Capre, a entrada em cena dos programas de pós-graduação das grandes universidades, a criação dos cursos de graduação plena, a formação da SBC e seu papel decisivo na consolidação de um modelo curricular hegemônico com a edição de currículos de referência e uma aliança com o Ministério da Educação.

Em outra vertente complementar, o texto reflete criticamente sobre os efeitos da organização universitária, especialmente as públicas, onde a estrutura departamental contribui para a preservação de currículos uni-disciplinares e onde a burocracia e os critérios de avalição inibem uma maior dedicação à melhoria do ensino de graduação.

\section{Seguindo o fio da meada}

Computadores foram introduzidos no Brasil no inicio da década de 1960. Tratava-se de conhecimento novo, ainda incipiente mesmo nas melhores universidades. Dado o grande interesse dos fabricantes internacionais em comercializar estes equipamentos no Brasil, estes tomaram a si a tarefa de formar os primeiros profissionais, através da criação de centros de treinamento próprios.

Os novos profissionais precisavam dominar o entendimento e a operação de máquinas extremamente complexas antes que fosse possível desenvolver qualquer aplicação útil. Isso levou a que a profissão fosse constituída principalmente por pessoas com formação em ciências naturais e engenharia, muito embora a maioria dos problemas tratados por computadores fossem tipicamente da área de humanas (administração, finanças, faturamento) e suas soluções afetassem o trabalho e o relacionamento de pessoas e organizações. $\mathrm{O}$ treinamento oferecido pelos fabricantes enfatizava a compreensão do funcionamento da máquina e a capacidade de dominar linguagens de programação baseadas em lógica.

No início da década de 1970 já existiam no Brasil organizações de grande porte com tradição na utilização de computadores, como Serpro, Petrobrás e as concessionárias de serviços públicos. Possuíam corpo técnico experiente, formado pelos fabricantes, mas já se sentiam capazes de formar seus próprios quadros, através de programas internos de treinamento. Típicos desse período foram os programas como os do Centro Nacional de Treinamento do SERPRO que recrutavam engenheiros, matemáticos, físicos e até administradores recém-formados, para transformá-los em analistas de sistemas ao longo de um curso intensivo de um ano [Lobato 1982,p.61].

Com a expansão acelerada do uso de computadores para atividades de processamento de dados, o governo cria em 1972, no Ministério do Planejamento, a CAPRE Coordenação das Atividades de Processamento Eletrônico. Entre as suas atribuições está a de formular um Programa Nacional de Ensino de Computação, visando suprir em prazo relativamente curto a forte demanda prevista de profissionais capacitados para suprir o mercado de trabalho em processamento de dados administrativos nos próximos anos. Com o apoio do MEC e de várias universidades, a CAPRE organiza com bastante 
sucesso o Projeto 19 para a criação de cursos de curta duração (máximo de 3 anos), denominados Cursos Superiores de Tecnologia em Processamento de Dados, também conhecidos como cursos de graduação curta. Cursos estes, por definição, destinados a formar profissionais capacitados a desenvolver aplicações de computadores de complexidade pequena e média, que respondiam então pela grande maioria da demanda de pessoal.

É interessante notar que o currículo e materiais didáticos dos cursos de tecnologia foram preparados por equipes convocadas nas universidades, sob a coordenação da CAPRE, mas procurando ouvir as sugestões e críticas dos empregadores dos futuros egressos, como mostra esse extrato de uma reunião realizada em 28/4/1974 em São Paulo, que envolveu 45 organizações entre a CAPRE, empresas e instituições de ensino, públicas e privadas (grifos nossos):

"A agenda dessa reunião constava de duas fases: ... a segunda, pela
tarde ... com a finalidade de detetar os desejos das empresas com
relação ao perfil do profissional a ser formado pelos cursos do
Projeto 19 em processamento de dados... Para auxiliar e objetivar a
formação do profissional voltada para as necessidades do mercado,
o Prof. Ziaviani aponta algumas atitudes que são da maior
importância: ...- debates e sugestões das empresas sobre o currículo
adotado." [CAPRE 1974b]"

O currículo resultante que foi aprovado pelo Conselho Federal de Educação, inclui matérias de tecnologia (formação e especialização em programação e uso de computadores) mas também dá espaço razoável para matérias que preparam o egresso para atuar profissionalmente, incluindo Economia e Finanças, Noções de Direito, Inglês e outras "visando o aprimoramento sócio-cultural do futuro profissional em processamento de dados" [CAPRE, 1974a].

\section{As universidades entram em cena - nasce a SBC}

As universidades, no começo apenas as maiores, começaram a atuar em computação principalmente pela via da pós-graduação. Após a implantação do regime militar em 1964, uma forte ênfase foi dada ao desenvolvimento em ciência e tecnologia. Os primeiros programas foram criados na UFRJ (COPPE 1968), PUC-Rio (1968), USP, Unicamp, UFMG, UFRGS (1973) e outras. O governo fomentou a formação de doutores no exterior com bolsas de estudo custeadas pelo Fundo de Desenvolvimento Tecnológico do Banco Nacional de Desenvolvimento Econômico (FUNTEC/BNDE). Em meados da década de 1970 já retornavam ao Brasil vários mestres e doutores em ciência e engenharia de computação.

Com o retorno dos primeiros pesquisadores, os programas de pós-graduação ganham impulso e em poucos anos esse contingente relativamente pequeno começa a se articular como um grupo com interesses comuns, voltados para a pesquisa científica, o ensino, e o desenvolvimento de computadores e da computação no Brasil, $\mathrm{Na}$ política, há um movimento em busca do apoio do governo para a implantação de computadores nas universidades e para uma indústria de computadores genuinamente brasileira. Para promover essas ideias, a comunidade de pesquisa organiza a realização dos Seminários de Computação nas Universidades (SECOMU), cuja primeira edição se dá em 1971, no 
Rio Grande do Sul, organizado por professores da PUC-Rio e com o apoio do Conselho de Reitores (CRUB) [Dantas 1988,p.88]. Na pesquisa e desenvolvimento, os pesquisadores organizam em janeiro de 1974 o primeiro SEMISH - Seminário sobre o Desenvolvimento Integrado de Software e Hardware, sediado pelo Curso de Pósgraduação em Ciência da Computação da UFRGS [Oliveira 2016]. É possível inferir que, nesse momento, a prioridade seria a tecnologia, e não o que os atores sociais fariam com ela.

Data desse período os projetos de criação de cursos de graduação plena em computação nas grandes universidades. A UFRJ, UFMG, PUC-Rio e UFRGS criam seus cursos de graduação, a exemplo das iniciativas pioneiras da Unicamp e da UFBA em 1969. Esses cursos, com denominações diversas como Ciência da Computação, Engenharia da Computação e Informática, duração de 4 a 5 anos, e currículos semelhantes, foram idealizados por pesquisadores e buscavam formar egressos com forte conteúdo matemático, científico e tecnológico. Houve desde o início um distanciamento claro entre os objetivos desses novos cursos e aqueles dos cursos de Tecnologia em Processamento de Dados. Os cursos de graduação plena enfatizavam o estudo dos fundamentos da ciência da computação e das tecnologias dos computadores, e abriam possibilidades para seus alunos prosseguirem tanto em estudos avançados de pósgraduação, como para atuarem em projeto e desenvolvimento de aplicações e na nascente indústria brasileira de computadores dos anos 70 .

Em 1978, em uma reunião histórica do SEMISH realizada na UFRJ, a comunidade acadêmica de computação considerou-se madura o suficiente para propor a criação de uma nova sociedade científica. Nascia assim a Sociedade Brasileira de Computação. [Cabral 2008,p.17]. O SEMISH e o SECOMU, antes eventos independentes, passam a integrar o Congresso Anual da SBC.

\section{A SBC atua na formação}

A SBC logo se preocupou com a rápida proliferação de cursos de graduação em computação que ocorria em todo o país nos anos 80, incluindo os de curta duração, motivada pela crescente procura de profissionais. Havia uma percepção de que muitos poderiam ser de qualidade duvidosa. Como relata Maria Izabel Cabral, os congressos anuais passaram a incluir uma reunião geral de coordenadores de cursos de graduação a partir de 1986, visando fomentar o debate sobre a formação e os currículos, e obter um consenso sobre o que deveria ser uma formação em computação. Em 1987, a sociedade organizou a edição de um catálogo dos cursos em todo o país, e lançou a iniciativa de elaborar um currículo de referência. Em 1990, no congresso de Vitória, a SBC constituiu a sua primeira Comissão de Ensino, embrião da atual Diretoria de Educação. A comissão, após diversas consultas à comunidade, e com apoio financeiro da Secretaria de Ensino Superior do MEC (SESu/MEC) produziu o Currículo de Referência que obteve sua aprovação na reunião de coordenadores de cursos realizada no congresso de 1991, referendada pela Assembleia Geral, em Santos, SP. [Cabral 2008, p.37].

O CR91, como ficou conhecido, reflete o pensamento dominante da comunidade acadêmica de computação das grandes universidades públicas e confessionais que constituía o núcleo da SBC. Desenvolvido por uma comissão composta por especialistas doutores em computação, e sem consulta a representantes do mercado empregador, 
como havia feito a Capre, não estranha que o currículo se concentre nas matérias de ciência e tecnologia, e dedique atenção marginal a questões humanas e sociais, sejam culturais, políticas, legais, organizacionais, psicológicas, econômicas ou filosóficas, que certamente fariam parte do universo dos egressos desses cursos. Para atender a essas questões, o CR91 apenas menciona, na seção referente aos Objetivos dos Currículos, que "Matérias de cultura geral devem ser incorporadas para prover uma formação humanística" e "Matérias de outras áreas podem ser introduzidas com vistas a formar pessoal especializado para participar na solução computacional de problemas que afetam cada uma dessas áreas". Direito, Economia e Administração apenas são sugeridas como matérias complementares [SBC 1991].

De fato, uma análise das matérias recomendadas pelo CR91 para cursos de Ciência da Computação e Engenharia de Computação mostra que aquelas consideradas essenciais incluíam: Álgebra, Lógica Matemática, Cálculo Diferencial e Integral, Álgebra Linear, Probabilidade e Estatística, Análise Numérica, Teoria da Computação, Linguagens Formais e Autômatos, Análise de Algoritmos, Máquinas Sequenciais e Combinacionais, Arquitetura e Organização de Computadores, Software Básico, Algoritmos e Estruturas de Dados, Paradigmas de Linguagens de Programação, Paralelismo, Banco de Dados, Sistemas Operacionais, Compiladores, Redes e Transmissão de Dados, Sistemas Distribuídos, Engenharia de Software e Projeto de Sistemas Digitais [SBC 1991].

Fica claro que neste primeiro currículo de referência a SBC postulava uma formação sem conexão com as ciências humanas e sociais para os futuros profissionais que teriam a missão de idealizar e produzir os sistemas computacionais que iriam influenciar profundamente as relações humanas e organizacionais do país nas próximas décadas.

O CR91 entra em cena como um forte aliado da visão tecnocientífica da formação em computação. Amplamente distribuído para todas as universidades com a chancela do porta-voz da comunidade científica, o CR91 tornou-se efetivamente um "ponto de passagem obrigatória" no sentido atribuído por Bruno Latour: "o fato [ou artefato] construído se torna indispensável" [Latour 2000, p.218]. De fato, a partir da aprovação, o currículo de referência passou a ser aceito sem maiores discussões pelos proponentes de novos cursos, o que pode ter contribuído para inibir visões alternativas de como formar os profissionais de computação.

A SBC continuou a influenciar fortemente a formação universitária em computação ao longo da década de 90. Em 1993, o Prof. Daltro Nunes, presidente da Comissão de Ensino, propôs a realização de um workshop para estender a toda a comunidade os debates que já ocorriam nas reuniões de coordenadores de cursos de computação durante o congresso anual. O Workshop sobre Educação em Computação / Informática foi realizado nos dias 7 e 8 de setembro de 1993, em Florianópolis, marcando o início de uma série que continua até hoje [Cabral 2008, p.37]. Na pauta, discussão sobre currículo de referência e infra-estrutura necessária para cursos de graduação.

Uma aliança tácita entre a SBC e a Secretaria de Ensino Superior do MEC (SESu) levou à formação da Coordenação de Especialistas de Ensino de Computação e Informática (CEEInf) da Diretoria de Ensino Superior da SESu/MEC, em 1994, que foi desde logo constituída por membros atuantes da SBC sob a liderança de Daltro Nunes [CEEInf, s/ data]. À CEEInf coube diversas missões que impactaram na evolução dos cursos de graduação em todo o país, especialmente os novos que iam sendo cridos em todas as 
regiões: conceituar e produzir os primeiros Padrões de Qualidade para avaliação dos cursos da área de Computação e Informática, organizar as comissões de avaliação dos cursos, homologar as avaliações e, mais adiante, coordenar os trabalhos para elaboração das primeiras Diretrizes Curriculares para a área em 1999, sempre em sintonia com a SBC. Paralelamente, a Comissão de Ensino, e sua sucessora, a Diretoria de Educação, continuaram a rever as recomendações curriculares, produzindo os currículos de referência de 1996 [SBC 1996], de 1999 [SBC 1999], e de 2005 [SBC 2005], as Diretrizes Curriculares de 1999 [BRASIL 1999] e, mais recentemente, as Diretrizes Curriculares de 2012 [BRASIL 2012].

Os Padrões de Qualidade embutiram a essência do CR91 no quesito currículo. As comissões nomeadas pela SESu/MEC para verificação e avaliação dos cursos de graduação eram orientadas pela CEEInf para exigir a conformidade com a essência do currículo de referência. A CEEInf, em parceria com a SBC, contribuiu decisivamente para a consolidação dos WEI's, e estimulou a criação dos Cursos de Qualidade a partir de 1999, que passaram a ser realizados desde então em conjunto com os WEI's.

O CR96, aprovado no Congresso de agosto de 1996, em Recife, introduz as matérias de Interfaces Homem-Máquina, no tópico Tecnologias da Computação, reconhece que o egresso deve possuir, entre as suas características, uma "formação humanística permitindo a compreensão do mundo e da sociedade" e inclui no tópico Contexto Social e Profissional as matérias de Computadores e Sociedade e de Formação de Empreendendores de Informática, as quais, no entanto, não são incluídas entre as rotuladas de "Importantes".

O CR99, para cursos de Computação e Informática, procurou ficar "em sintonia" com as Diretrizes Curriculares de 1999, ambas abrangendo tanto cursos como atividade fim e como atividade meio. Enquanto o CR99 foi elaborado no âmbito da Diretoria da Educação da SBC, e aprovadas no WEI de julho de 1999, as Diretrizes, elaboradas pela CEEInf, foram finalizadas em março do mesmo ano. Ambas tiveram a colaboração intensa da comunidade acadêmica de computação, com muita interseção. No item "Formação Humanística" das diretrizes aparecem pela primeira vez as matérias de História da Ciência da Computação (porque não da computação em geral?), Ética, Sociologia e Filosofia, além de Computadores e Sociedade e Empreendedorismo. Não há sugestão de um percentual de horas para essa área de formação, nem como devem se relacionar com o restante do curso, apenas a recomendação que "As disciplinas devem cobrir essa matéria de forma geral".

O CR2005, que se ateve aos cursos de Ciência e Engenharia (atividade fim), indica que o perfil dos egressos, em seus "Aspectos Gerais", deve incluir uma "formação humanística permitindo a compreensão do mundo e da sociedade", além de "habilidades de trabalho em grupo e de comunicação e expressão". E em seus "Aspectos Ético-Sociais" assinala que os egressos devem "Respeitar os princípios éticos da área de computação" e "Ter uma visão humanística crítica e consistente sobre o impacto de sua atuação profissional na sociedade”. O currículo inclui um núcleo de matérias de Contexto Social e Profissional que lista 14 matérias. É interessante observar que há uma diretiva de recomendar 30 créditos para esse núcleo, de um total de 160 a 200 créditos previstos para todo o curso, ou cerca de $15 \%$. 
Finalmente, as novas Diretrizes de 2011/12 resultaram de uma extensa revisão das Diretrizes de 1999 (nunca homologadas formalmente) realizada por um grupo de trabalho formado por membros tradicionais da SBC e da Diretoria de Educação, coordenado pelo incansável Daltro Nunes. Elas expressam uma conscientização bem maior sobre a necessidade de se ampliar a formação nos cursos de computação para além dos conteúdos técnicos e científicos. Em um extenso trecho dedicado à Formação Humanística e Social o texto reconhece que "A Computação permeia praticamente todas as atividades humanas, incluindo trabalho, lazer, saúde e comunicação, cabendo aos profissionais da Área a responsabilidade pelo desenvolvimento de soluções, ferramentas e processos coerentes com a moral, bons costumes, valores éticos e interesse social, e que também busquem o bem-estar do homem e o avanço tecnológico". E mais, considera indispensável os estudos de História da Computação, do empreendedorismo, das questões éticas, dos impactos da automação na sociedade, da Filosofia e da Sociologia, incluindo mudanças na organização do trabalho provocadas pelas inovações tecnológicas e a "compreensão e o questionamento do mundo tecnológico e do mundo sociocultural que o circunda". E conclui enfatizando que "O estudo integral da Computação transcende as questões meramente técnicas, exigindo a compreensão do processo de construção do conhecimento”. [BRASIL 2012]

\section{Obstáculos a uma formação interdisciplinar}

Nas seções anteriores apresentamos a emergência da formação típica em ciência e engenharia da computação nas nossas universidades voltada quase que totalmente para os estudos de ciência e tecnologia. Apesar da grande maioria dos egressos desses cursos se tornarem profissionais responsáveis por desenvolver artefatos e sistemas com potencial de afetar diretamente as atividades humanas, muitos currículos atuais ainda permanecem essencialmente semelhantes em conteúdo aos primeiros currículos de décadas atrás. Vimos como as diretrizes e recomendações curriculares foram aos poucos abrindo espaço para uma formação mais interdisciplinar ao reconhecer o caráter multifacetado do profissional de computação.

Existem, no entanto, outros obstáculos para que transformações nesse sentido sejam de fato realizadas.

A Reforma Universitária de 1968 [BRASIL 1968]instituiu a descentralização das competências acadêmicas, ao criar os institutos e departamentos especializados nas universidades públicas [Martins 2009]. Essa forma de organização acadêmica horizontalizada procurou reunir os pares em estruturas homogêneas de conhecimento, reunindo pesquisa e ensino, visando uma maior interação e produtividade na produção acadêmica. Na forma anterior verticalizada, as unidades se organizavam pelas suas finalidades de formação: cada escola reunia no seu corpo docente especialistas de diversas áreas para organizar o ensino dos seus alunos. As escolas de engenharia, por exemplo, contratavam matemáticos, físicos, químicos, professores de línguas, filosofia, e o que mais necessitassem. Com a reforma, cada área de conhecimento ficou mais compartimentalizada em seu departamento.

O caso específico da computação é emblemático, pois os primeiros cursos de computação já foram criados na nova estrutura, que permanece até os nossos dias. 
Docentes contratados por critérios de qualificação para a pesquisa acadêmica em determinadas especialidades são reunidos em departamentos de computação, e passam a responder na prática pelos currículos dos cursos. Muitos passam diretamente de uma formação puramente acadêmica em computação para a atividade docente, sem terem tido experiência profissional e conhecimento do mercado de trabalho onde seus alunos irão atuar. É nesse ambiente que são discutidos e modificados os currículos dos cursos de graduação. Ambiente que é naturalmente pouco permeável a promover uma formação integral do aluno para além das especialidades do grupo, levando os currículos a continuarem mais ou menos estáveis.

Esses fatores contribuem para inibir mudanças em profundidade nos currículos, onde o ensino das disciplinas reflete uma visão idealizada da ciência e da tecnologia como conhecimento universal, neutro e desinteressado, que são apresentadas sem perspectiva histórica ou local, e sem relacionamento com questões culturais, econômicas e sociais. Não há preocupação em estimular o pensamento crítico dos alunos e formar juízos de valor sobre as consequências do uso das tecnologias de computação nas diversas esferas da sociedade. Disciplinas como Computadores e Sociedade são uma exceção, mas representam uma fração muito pequena da formação e são ministradas em geral sem relação com as demais disciplinas do currículo.

Uma mudança dessa natureza requer tempo e esforço. A falta de autoridade real dos coordenadores de curso, aliada ao baixo incentivo dessa função para o progresso na carreira docente, dificultam e inibem iniciativas mais inovadoras de reforma curricular, que envolvem gerir conflitos de interesse, obter o apoio dos pares, articulações com outras unidades e dedicação e esforço continuado por vários meses. Por outro lado, as agências de fomento e os critérios internos de progressão na carreira contribuem para reduzir o interesse dos docentes pelo aperfeiçoamento do ensino de graduação, ao valorizar muito mais os resultados da pesquisa acadêmica.

\section{Conclusões}

A contribuição dos estudos de ciência, tecnologia e sociedade (CTS) neste debate se dá pela exposição das circunstancias e das redes de relacionamentos que levaram à atual situação do ensino de computação nas universidades. Os Estudos CTS permitem colocar em questão práticas estabelecidas que, de tão repetidas, são percebidas como naturalizadas, como resultantes de uma evolução natural que não poderia ter sido diferente.

Os Estudos CTS não produzem diretamente, e nem necessariamente, propostas de caminhos alternativos. O propósito é oferecer uma narrativa que apresente um olhar descompromissado, que estimule a reflexão e permita perceber outros caminhos possíveis a partir do entendimento das circunstâncias e contingências, envolvendo pessoas, interesses, regulamentos, instituições e vários outros atores, que levaram à presente situação. 


\section{Referências}

BRASIL (1968) Lei $\mathrm{n}^{\circ}$ 5.540, de 28 de Novembro de 1968 (Reforma Universitária), disponível em <http://www2.camara.leg.br/legin/fed/lei/1960-1969/lei-5540-28novembro-1968-359201-publicacaooriginal-1-pl.html>. Acesso em 03/09/2013.

BRASIL. (1999) MEC/ Secretaria de Educação Superior, Departamento de Políticas do Ensino Superior. Comissão de Especialistas de Ensino de Computação e Informática. Diretrizes curriculares de cursos da área de computação e informática. Brasília: MEC, Disponível em: < http://www.inf.ufrgs.br/ecp/docs/diretriz.pdf $>$. Acesso em: $10 / 5 / 2016$.

BRASIL (2012) MEC/CNE/CES Diretrizes Curriculares Nacionais para os cursos de graduação em Computação. Parecer CNE/CES no. 136/2012 Disponível em http://portal.mec.gov.br/index.php?option $=$ com_docman\&view $=$ download\&alias $=112$ 05-pces136-11-pdf\&category_slug=julho-2012-pdf\&Itemid=30192, acesso em $10 / 12 / 2015$.

Cabral, M.I.C. et al. (2008) A Trajetória dos Cursos de Graduação da Área de Computação e Informática: 1969-2006. Rio de Janeiro: Editora SBC.

Capre (1974a) Curso Técnico de Nível Superior em Processamento de Dados, Boletim Informativo, v.2(2), 42-50, abr/jun 1974.

Capre (1974b) Reunião do Projeto 19 com as Empresas, Boletim Informativo, v.2(3), 20-29, jul/set 1974.

CEEInf (s/data) DPES/SESu/MEC Comissão de Especialistas de Ensino de Computação e Informática. Disponível em: http://www.inf.ufrgs.br/mec/ceeinf.comissao.html, acesso em $2 / 2 / 2016$

Dantas, V. (1988) Guerrilha Tecnológica - A Verdadeira História da Política Nacional de Informática. Rio de Janeiro: LTC-Livros Técnicos e Científicos Editora Ltda., 1988.

Latour, B. (2000) Ciência em Ação: como seguir cientistas e engenheiros sociedade afora. São Paulo: Editora UNESP.

Latour, B. (2007) Reassembling the Social-An Introduction to Actor-Network-Theory. New York: Oxford University Press.

Lobato, W.S. (1982) SERPRO -Uma crônica de 18 anos, 1982. Publicação avulsa, disponível em https://www.serpro.gov.br/sala-deimprensa/conteudo/p_publicacoes/serpro-18-anos.pdf/@@download/file/livro-18anos.pdf, acesso em 19/05/2016.

Martins, C.B. (2009) A reforma universitária de 1968 e a abertura para o ensino superior privado no Brasil. Educação e Sociedade, Campinas, vol. 30, n. 106, p. 15-35, jan./abr. 2009. Disponível em http://www.scielo.br/pdf/es/v30n106/v30n106a02. 
Oliveira, J.P.M. (2016) I SEMISH -1974. Site do Prof. Palazzo. Disponível em http://www.palazzo.pro.br/Wordpress/i-semish-1974/, acesso em 19/05/2016.

SBC (1991) Currículo de Referência da SBC para Cursos de Graduação Plena em Computação 1991. Diretoria de Educação, Sociedade Brasileira de Computação, junho de 1991. Disponível em: http://homepages.dcc.ufmg.br/ bigonha/Cr/cr91.html, acesso em 17/2/2015.

SBC (1996) Currículo de referência da SBC para cursos de graduação em computação, versão 1996 - Parte 1, Diretoria de Educação, Sociedade Brasileira de Computação. Disponível em: http://homepages.dcc.ufmg.br/ bigonha/Cr/crf96.html, acesso em 24/5/2016.

SBC (1999) Currículo de referência da SBC para cursos de graduação em computação, versão 1999 - Parte 1, Diretoria de Educação, Sociedade Brasileira de Computação. Disponível em: http://lad.dsc.ufcg.edu.br/ec/cr99.pdf, acesso em 24/5/2016.

SBC (2005) Currículo de Referência da SBC para Cursos de Graduação em Bacharelado em Ciência da Computação e Engenharia de Computação., Proposta versão 2005. Diretoria de Educação, Sociedade Brasileira de Computação. Disponível em http://www.sbc.org.br/documentos-da-sbc/summary/131-curriculos-de-

referencia/760-curriculo-de-referencia-cc-ec-versao2005, acesso em 24/5/2016. 